\title{
Modeling the LAGO's detectors response to secondary particles at ground level from the Antarctic to Mexico
}

\author{
R. Calderón-Ardila ${ }^{2,3}$, A. Jaimes-Motta ${ }^{1}$, J. Peña-Rodríguez ${ }^{* 1}$, C. Sarmiento-Cano ${ }^{2,3 \dagger}$ \\ M. Suárez-Durán ${ }^{1,3,4}$, A. Vásquez-Ramírez ${ }^{1}$, for the LAGO Collaboration ${ }^{5}$ \\ ${ }^{1}$ Escuela de Física, Universidad Industrial de Santander, Bucaramanga, Colombia \\ ${ }^{2}$ Universidad Nacional de San Martín (UNSAM), Buenos Aires, Argentina \\ ${ }^{3}$ Instituto de Tecnologías en Detección y Astropartículas (ITeDA), Buenos Aires, Argentina \\ ${ }^{4}$ Departamento de Física y Geología, Universidad de Pamplona, Pamplona, Norte de Santander, \\ Colombia \\ ${ }^{5}$ ht $t$ : / / Iagopro ject. net/, see the full list of members and institutions at \\ http://lagoproject.net/collab.html \\ E-mail: christian.sarmientoditeda.cnea.gov.ar
}

\begin{abstract}
The Latin American Giant Observatory (LAGO) is an observatory spanning over Latin America in a wide range of latitudes with different rigidity cut offs and atmospheric depths. The motivation of the Observatory is to study, using Water Cherenkov Detectors (WCD), the atmospheric radiation and the space weather through the measurement of secondary particles produced during the interaction of astroparticles with the atmosphere. Here, we present the methodology for estimating the number of Cherenkov photons detected by the photo-multiplier tube in a WCD in any geographical position. To do this, the secondary particle flux of cosmic rays is calculated and a geomagnetic field correction is applied. The CORSIKA and MAGCOS programs are used. In addition, the outcome of the simulation is used as an input to the Geant4 program in order to mimic the response of the detector. As a result, the distribution of photo-electrons is obtained for the total particle flux, where its behavior resembles the characteristic signals of this kind of detector and could be used to calibrate the system. This methodology was applied for five LAGO detector sites, located at different latitudes and altitudes above sea level, between Chile and Mexico.
\end{abstract}

36th International Cosmic Ray Conference -ICRC2019-

July 24th - August 1st, 2019

Madison, WI, U.S.A.

* Speaker.

Corresponding author 


\section{Introduction}

The detection of secondary particles at the ground level is one of the techniques used in astroparticle physics. This technique can be used to study transient events such as gamma-ray burst or Forbush decreases [1], or in applications such as muon radiography [2], known also as muography. A detailed knowledge of how the secondaries are produced in the atmosphere and how they reach a geographical position is needed for this investigations.

Currently, computational tools as CORSIKA [3] and FLUKA [4] allow the estimation of the nominal flux of those particles that arrive to the ground (background radiation). This simulation is based on the measured spectra of Galactic Cosmic Rays (GCR). The estimation allows for the calculation of the detector response to the background radiation using computational models, such as Geant4 [5], and the prediction of the effect that GCR fluctuations may have on it. The integration of these computational tools makes it possible to design new observatories for GCR, and to apply models to understand how a fluctuation on the measured signal is correlated, or not, with a perturbation on the nominal GCR flux; for instance, gamma ray burst or solar-related activity.

Detecting background radiation and using it to study astroparticles and geophysical phenomena is one of the the main goals of the Latin American Giant Observatory (LAGO). This is an extended observatory of water Cherenkov detectors (WCDs) at continental scale. It covers a large range of geomagnetic rigidity cutoffs and atmospheric absorption depths [6], (see Figure1). In this sense, LAGO is promoting training and research in astroparticle physics in Latin America, covering three main areas: search for the high-energy component of gamma ray bursts at high altitude sites, space weather phenomena, and background radiation at the ground level [7, 8].

We developed the full computational framework to estimate the signals expected at the LAGO detector sites. This framework, which includes the effect of the geomagnetic field on the GCR propagation [8], is composed by a set of individual tools, collectively named as ARTI ${ }^{1}$.

In this document, Section 2 introduces the method implemented by LAGO to estimate the nominal background radiation, including the correction by the geomagnetic field, at five representative locations, referenced by latitude and altitude as follows (Latitude, Altitude): Ciudad de Guatemala, Guatemala (14.63 ${ }^{\circ}, 1490 \mathrm{~m}$ a.s.1.); Bucaramanga, Colombia ( $7.14^{\circ}, 956 \mathrm{~m}$ a.s.1.); Quito, Ecuador $\left(-0.2^{\circ}, 2800 \mathrm{~m}\right.$ a.s.1.); Chacaltaya, Bolivia $\left(-16.35^{\circ}, 5240 \mathrm{~m}\right.$ a.s.1. $)$ and La Serena, Chile $\left(-29.90^{\circ}, 28 \mathrm{~m}\right.$ a.s.1.). The results of the estimated signal for a standard WCD, modeled using Geant4 code, are presented for each of these positions in Section 3. Finally, in Section 4, the final remarks and future perspectives are discussed.

\section{Estimation of Cosmic Background Radiation at The Ground Level}

The flux of secondary particles at the ground was calculated for each LAGO site, following the method developed in [8]. In this method, the GCR flux $(\Phi)$ is calculated at an altitude of $112 \mathrm{~km}$ a.s.l. Here, $\Phi$ is considered as

$$
\Phi\left(E_{p}, Z, A, \Omega\right) \simeq j_{0}(Z, A)\left(\frac{E_{p}}{E_{0}}\right)^{\alpha\left(E_{p}, Z, A\right)},
$$

\footnotetext{
${ }^{1}$ http://wiki.lagoproject.net/index.php?title=ARTI
} 

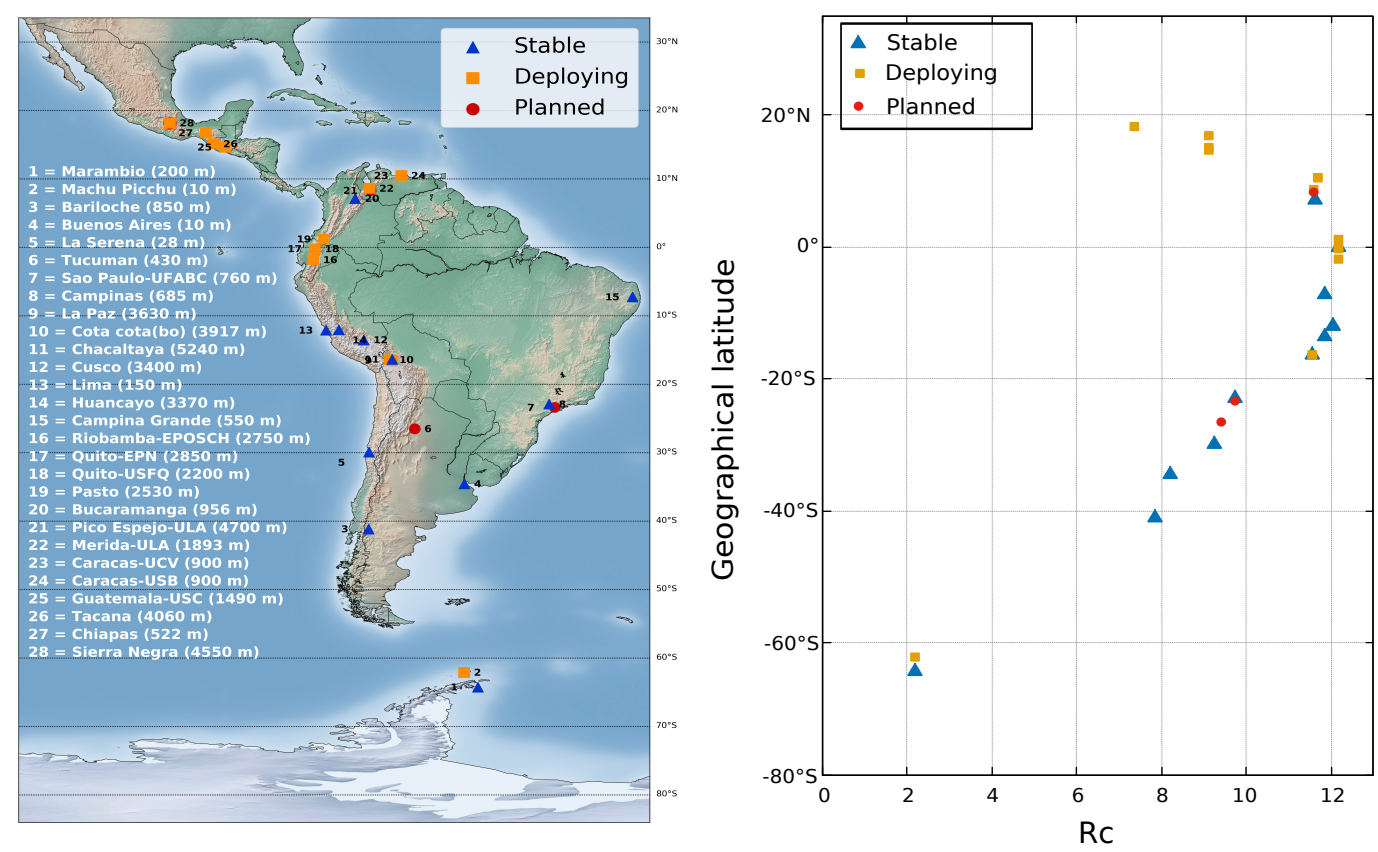

Figure 1: (left) Geographical distribution and altitudes of the Latin American Giant Observatory water Cherenkov detectors: the ones in operation are represented with blue triangles, orange squares are used for those in deployment and the planned sites are indicated in red circles. (right) Vertical rigidity cutoff at each Latin American Giant Observatory site.

where $E_{p}$ is the energy of the particle, $\alpha\left(E_{p}, Z, A\right)$ is considered constant with respect to the energy, i.e. $\alpha\left(E_{p}, Z, A\right) \approx \alpha(Z, A)$, from $10^{11} \mathrm{eV}$ to $10^{15} \mathrm{eV}$ [9], and $E_{0}$ has a value of $10^{12} \mathrm{eV}$.

In the first stage, ARTI uses CORSIKA to calculate the particles produced by the interaction of each GCR with the atmosphere [3]. Thus, we can estimate the expected flux of secondary particles at the detector level for each LAGO site.

To achieve this, the CORSIKA 76500 version was used, compiled with the following options: QGSJET-II-04,[10]; GHEISHA-2002; EGS4; curved and external atmosphere and volumetric detector. The IGRF-12 model provides the local geomagnetic field values, $B_{x}$ and $B_{z}$, required by CORSIKA to take into account the geomagnetic effects on the particles propagation in the atmosphere.

In this simulation, each secondary particle is tracked up to the lowest energy threshold that CORSIKA allows $\left(E_{s}\right)$, according to the type of the secondary. Currently, these threshold are $E_{s} \geq$ $5 \mathrm{MeV}$ for $\mu^{ \pm}$and hadrons (excluding $\pi^{0}$ ); and $E_{s} \geq 5 \mathrm{KeV}$ for $e^{ \pm}, \pi^{0}$ and $\gamma$. Since the atmospheric profile is a key factor for the production of secondary particles, and a parameter for CORSIKA, we have to set atmospheric MODTRAN profiles models [11] according to the geographical position of the LAGO sites: a tropical profile for Bucaramanga (BGA), Ciudad de Guatemala (GUA), Quito (UIO), La Serena (LSC) and Chacaltaya (CHA). In this way, we estimate the spectrum of secondary particles $(\Xi)$.

ARTI uses input parameters such as the city code (the IATA and/or ICAO airport code ${ }^{2}$ ), time length for the flux simulation, the magnetic field, energy range, type of primary particle and

\footnotetext{
${ }^{2}$ IATA: https://en.wikipedia.org/wiki/IATA_airport_code; ICAO: https://en.wikipedia.org/wiki/ICAO_airport_code
} 
the angular distribution to create a CORSIKA data file ${ }^{3}$, needed to perform the corresponding simulations, one for each GCR.

In this work, the flux $\Xi$ has been calculated for each of the five LAGO sites. Figure 2 shows examples of the results for the obtained spectra, for each type of secondary at CHA (5240 $\mathrm{m}$ a.s.l.) and LSC (28 $\mathrm{m}$ a.s.1.). As expected, there are less particles at low altitude due to atmospheric absorption. Namely, the flux at CHA is larger than the one at LSC for each type of secondary. Furthermore, the CORSIKA energy cuts $\left(E_{s}\right)$ over the respective types of secondaries can also be seen in the plots.

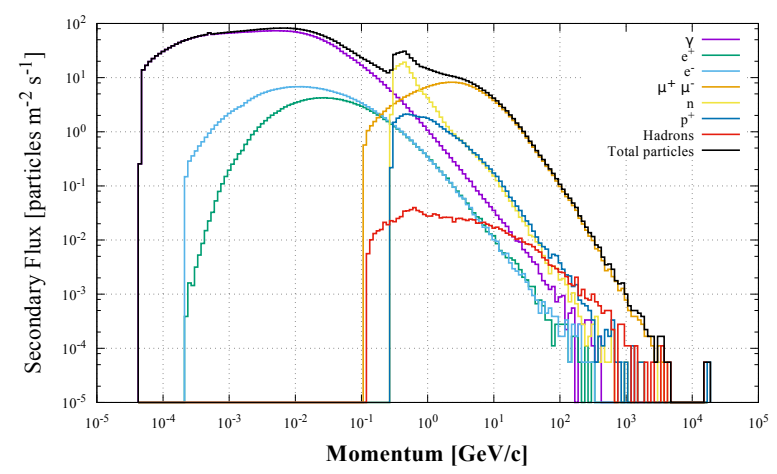

Chacaltaya

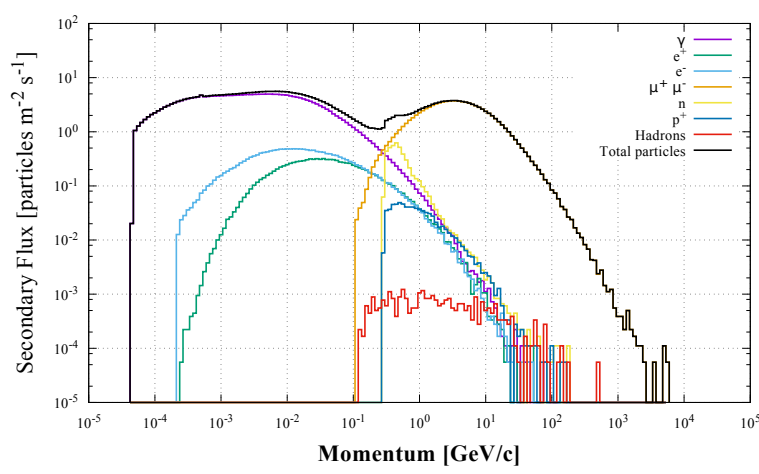

La Serena

Figure 2: Spectrum of the secondary particles at two LAGO sites: (left) Chacaltaya, Bolivia (5240 m a.s.1.); (right) La Serena, Chile (28 $\mathrm{m}$ a.s.1.). The comparison of the two plots put in evidence a difference of one order of magnitude (at $\sim 10^{-2} \mathrm{GeV} / \mathrm{c}$ ) in the total of secondaries (black line). At $\sim 3.5 \times 10^{-1} \mathrm{GeV} / \mathrm{c}$, the neutron component (yellow line) for Chacaltaya dominates over the $\mu^{ \pm}$ component (orange line), while for the La Serena estimation is the opposite. Since the LAGO detectors calibration is based on the muons, it is important to note that the prediction for the muon component is larger than for the $e^{ \pm}$(green an blue lines) ones at La Serena; meanwhile, at Chacaltaya, $e^{ \pm}$dominates with respect to $\mu^{ \pm}$due to atmospheric development of hadronic cascades.

\subsection{Cosmic Background Radiation at ground corrected by Geomagnetic Field}

Low energy GCRs $\left(E_{p} \lesssim 20 \mathrm{GeV}\right)$ trajectories are deflected by the Earth magnetic field (GF). The deflection is parametrized by the magnetic rigidity term $\left(R_{\mathrm{m}}\right)[12,13,14]$. For instance, transient solar phenomena, such as Forbush decrease (FD) events, change the GF lines, the flux at low energy and, therefore, the cosmic background radiation at the ground $[15,1,16]$. The FD events have been registered by different observatories using WCDs [17, 18, 19, 20]. In this sense, the LAGO Collaboration have developed the LAGO Space Weather (LAGO-SW) program [8], to study the variations in the flux of secondary particles at ground level and their relation to the heliospheric modulation of GCRs [8]. The GF effect on the flux $\Xi$ has been included in this work for each of the five LAGO sites, following the LAGO-SW method, i.e. ignoring the secondaries produced by GCRs that do not reach the respective location. This effect is the second component of the ARTI framework.

It is important to remark that this method builds a magnetic rigidity cutoff $\left(R_{\mathrm{C}}\right)$ as a function of the geographical latitude, longitude, altitude above sea level, the arrival direction $(\phi$ and $\theta)$ and a cumulative probability distribution function for the penumbra region, at it is explained in [8].

\footnotetext{
${ }^{3}$ ASCII file with all the parameters listed
} 
Table 1: Flux of cosmic background radiation at ground $\left(\Xi\left[\mathrm{m}^{-2} \mathrm{~s}^{-1}\right]\right)$ estimated at for five LAGO sites: Chacaltaya, Bolivia (CHA); Quito, Ecuador (UIO); Ciudad de Guatemala (GUA); Bucaramanga, Colombia (BGA); and La Serena, Chile (LSC). The flux for each secondary type is presented as follow: $e^{ \pm}$and $\gamma\left(\Xi^{\mathrm{EM}}\right) ; \mu^{ \pm}\left(\Xi^{\mu}\right)$; neutrons $\left(\Xi^{\mathrm{n}}\right)$; and all secondaries $\left(\Xi^{\mathrm{All}}\right)$. GE ${ }^{i}[\%]$ represents how bigger was the GF effect over each type of secondary (estimated as the percent difference with respect to the flux $\Xi$ without GF effect), with $i$ according to the $\Xi$ notation before.

The results for the estimated flux of cosmic background radiation at ground, including the GF correction, for the five LAGO sites are presented in Table 1 and Figure 3. Here, we can see a correlation between the flux $\Xi$ and the altitude, i.e., $\Xi$ increases with the altitude.

\begin{tabular}{|c|c|c|c|c|c|c|c|c|c|}
\hline $\begin{array}{c}\text { LAGO } \\
\text { site }\end{array}$ & $\begin{array}{c}\text { Alt } \\
{[\mathbf{m} \text { a.s.l.. }]}\end{array}$ & $\begin{array}{c}\Xi^{\mathrm{EM}} \\
{\left[\mathrm{m}^{-2} \mathrm{~s}^{-1}\right]}\end{array}$ & $\begin{array}{c}\mathbf{G E}^{\mathrm{EM}} \\
{[\%]}\end{array}$ & $\begin{array}{c}\Xi^{\mu} \\
{\left[\mathrm{m}^{-2} \mathrm{~s}^{-1}\right]}\end{array}$ & $\begin{array}{c}\mathbf{G E}^{\mu} \\
{[\%]}\end{array}$ & $\begin{array}{c}\Xi^{\mathrm{n}} \\
{\left[\mathrm{m}^{-2} \mathrm{~s}^{-1}\right]}\end{array}$ & $\begin{array}{c}\mathbf{G E}^{\mathrm{n}} \\
{[\%]}\end{array}$ & $\begin{array}{c}\Xi^{\text {All }} \\
{\left[\mathrm{m}^{-2} \mathrm{~s}^{-1}\right]}\end{array}$ & $\begin{array}{c}\mathbf{G E}^{\mathrm{All}} \\
{[\%]}\end{array}$ \\
\hline CHA & 5240 & 4030 & -15.4 & 231 & -12.5 & 150 & -81.3 & 4450 & -17.7 \\
\hline UIO & 2800 & 1073 & -9.87 & 147 & -7.48 & 35.0 & -60.0 & 1263 & -11.6 \\
\hline GUA & 1490 & 591.0 & -3.72 & 123 & -2.43 & 16.0 & -31.2 & 733.0 & -4.22 \\
\hline BGA & 956.0 & 424.0 & -5.42 & 109 & -3.66 & 9.00 & -44.4 & 544.0 & -5.88 \\
\hline LSC & 28.00 & 282.0 & -2.48 & 96.0 & -1.04 & 4.00 & -25.0 & 384.0 & -2.60 \\
\hline
\end{tabular}

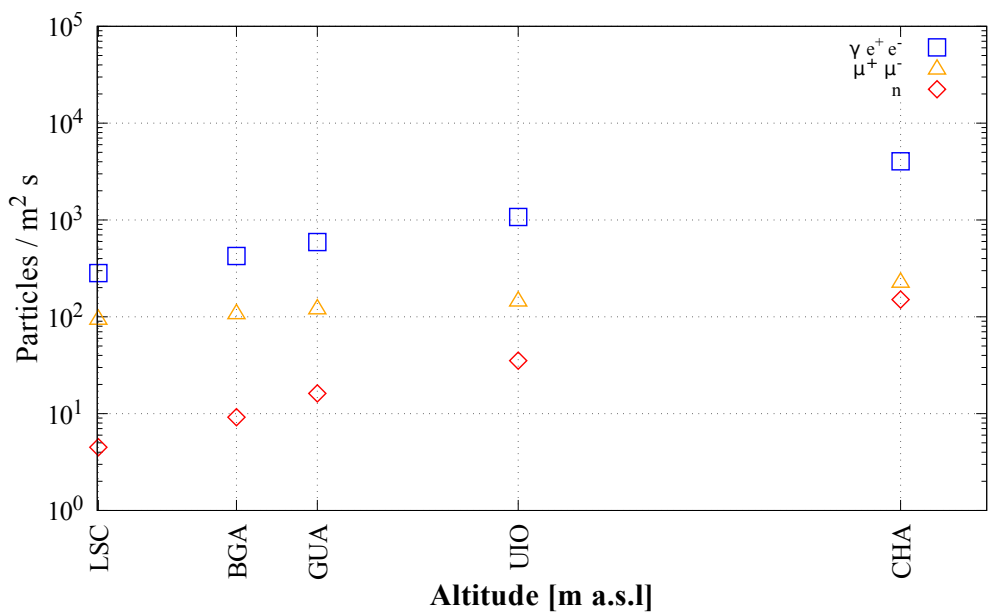

Figure 3: Flux $(\Xi)$ of cosmic background radiation at ground for each LAGO site and for the different components: electromagnetic in blue squares $\left(\gamma, e^{-}, e^{+}\right)$, muonic in yellow triangles $\left(\mu^{-}, \mu^{+}\right)$and neutrons in red diamonds (n). The flux is showed as a function of the altitude above sea level. On each component, a correlation between $\Xi$ and altitude is observed, i.e. $\Xi$, increases with the altitude as expected due to atmospheric absorption. 
of the cylinder [22]. LAGO-GD uses the estimated flux $\Xi$ as an input parameter, distributing the number of particles on a circular area $A$, just above the WCD, during a time $t$, always conserving the flux $\Xi$.

With any cylindrical configuration (radius and height), LAGO-GD models and estimates the signal produced by Cherenkov effect as the number of photo-electrons (pe) produced in the PMT device. A pe is produced according to the quantum efficiency $(\mathrm{QE})$ of the corresponding PMT (in this case, the QE from [23]). In this work, a standard LAGO WCD has been modeled with $1.05 \mathrm{~m}$ of radius and $0.90 \mathrm{~m}$ of height for all the sites. The geometry of the PMT (fully immersed in water) is taken as the photo-cathode surface with a semi-ellipsoid of semi-axis $(0.101 \mathrm{~m}, 0.101 \mathrm{~m}$, $0.065 \mathrm{~m})$.

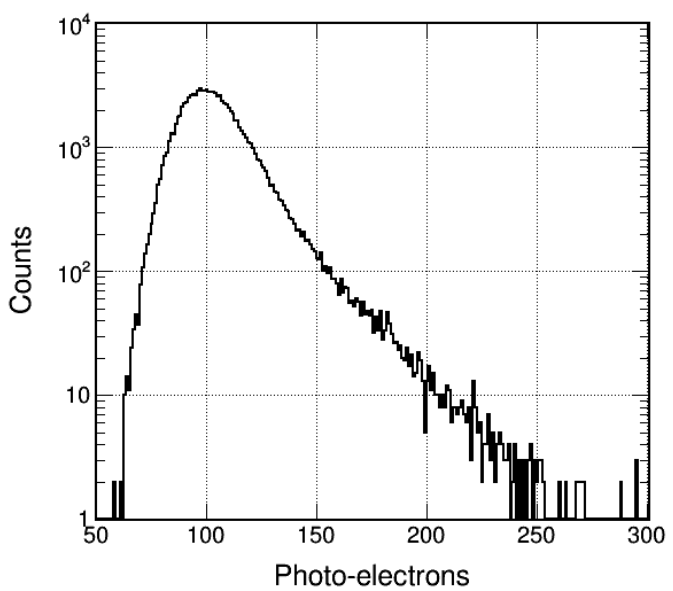

Figure 4: Distribution of the number of photo-electrons obtained for $10^{5} 3 \mathrm{GeV}$ VEM using LAGO-GD. The mode of 100 pe represents the unit of calibration (1 VEM), i.e. 100 pe corresponds to $\sim 180 \mathrm{MeV}$ of energy deposited.

The results obtained with LAGO-GD are presented according to the calibration process described in [24, 25]: converting the signal detected (in this case the number of pe) into physical units of energy deposited $\left(E_{\mathrm{d}}\right)$. A single high-energy muon impinging vertically at the center of the WCD, called Vertical Equivalent Muon (VEM) [24, 26], is the calibration unit defined as the average charge collected in the PMT.

In order to estimate the signal detected, the number of pe produced by VEM was calculated first. Figure 4 shows the distribution of pe obtained with LAGO-GD for $10^{5}$ VEMs of $3 \mathrm{GeV}$ of energy, where the most probable number of pe is $\sim 100$. This number corresponds to $E_{\mathrm{d}} \sim$ $180 \mathrm{MeV}$, with a muon stopping power in water of $2 \mathrm{MeV} / \mathrm{cm}$; thus, our unit of calibration is $1 \mathrm{VEM} \sim 100 \mathrm{pe} \approx 180 \mathrm{MeV}$.

The charge histograms obtained for Chacaltaya and La Serena are shown in the Figure 5, where the black curve represents the total contribution of all the particles detected with the WCD. Through the simulations, it is possible to estimate the response of the WCD to different components of the Extensive Air Showers (EAS), showed in different colors. It is remarkable that the main source of the first peak is the electromagnetic component (gammas, electrons and positrons) while the second peak is dominated by the muon component. Those particles travel more distance in water, producing more Cherenkov photons than the VEM. The rate of particles detected $\left(\Xi^{D}\right)$ and its 
contribution to the total energy deposited in the WCD are shown in the Table 2.
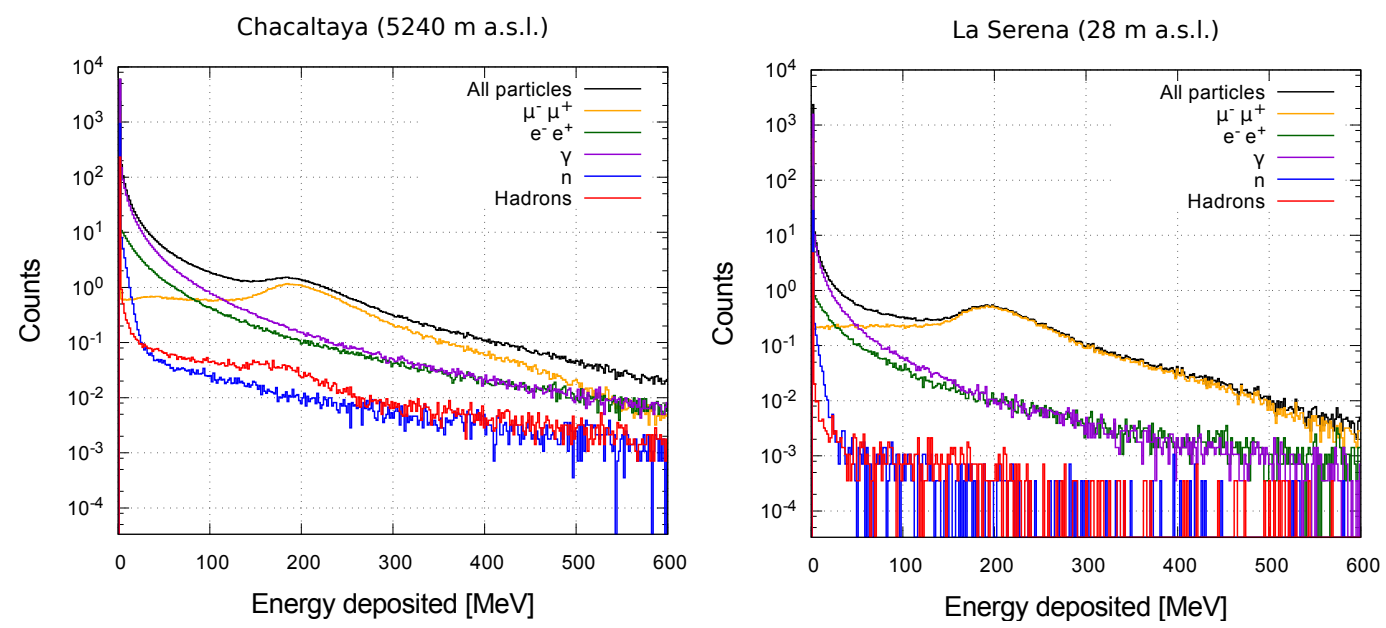

Figure 5: Charge histograms obtained for Chacaltaya (left) and La Serena (right) in an exposition time of 1 second. The black curves represent the total deposited energy and the colour ones represent the contribution of the EAS components.

Table 2: Rate of the secondary particles detected in the WCD for five LAGO sites, $\Xi^{D}$, and the energy deposited by the electromagnetic component, $E_{\mathrm{d}}^{\mathrm{EM}}$, muon component, $E_{\mathrm{d}}^{\mu}$, neutrons, $E_{\mathrm{d}}^{\mathrm{n}}$, and all the particles, $E_{\mathrm{d}}^{\mathrm{All}}$, from $\Xi^{D}$.

\begin{tabular}{|c|c|c|c|c|c|}
\hline LAGO Site & $\Xi^{D} \times 10^{3}\left[\mathrm{~m}^{-2} \mathrm{~s}^{-1}\right]$ & $E^{\mathrm{EM}}[\mathrm{GeV}]$ & $E^{\mu}[\mathrm{GeV}]$ & $E^{\mathrm{n}} \times 10^{-1}[\mathrm{GeV}]$ & $E^{\mathrm{All}}[\mathrm{GeV}]$ \\
\hline CHA & 1.80 & 1.49 & 0.22 & 0.49 & 1.77 \\
\hline UIO & 0.52 & 0.40 & 0.14 & 0.11 & 0.55 \\
\hline GUA & 0.31 & 0.22 & 0.11 & 0.05 & 0.34 \\
\hline BGA & 0.23 & 0.16 & 0.10 & 0.03 & 0.26 \\
\hline LSC & 0.17 & 0.11 & 0.09 & 0.01 & 0.20 \\
\hline
\end{tabular}

\section{Conclusions}

The ARTI framework, which we present here, allows us to estimate what would be the charge histogram for each site of LAGO. Therefore, those histograms can be compared with the data collected experimentally in order to calibrate the WCDs. In addition, this work agrees with the results presented in [27] where the relationship between the secondary particle flux and the height at which the detector is located was shown; see Figure 2. Finally, we were able to develop a tool that estimates the flux of secondary particles detected by a WCD in any geographic position and at any time of the year.

\section{Acknowledgment}

The LAGO Collaboration is very thankful to all the participating institutions and to the Pierre Auger Collaboration for their continuous support. In addition, some results presented in this paper 


\section{References}

[1] I. G. Usoskin, I. Braun, O. G. Gladysheva, J. R. Hörandel, T. Jämsén, G. A. Kovaltsov, and S. A. Starodubtsev. Forbush decreases of cosmic rays: Energy dependence of the recovery phase. Journal of Geophysical Research: Space Physics, 113(A7), jul 2008.

[2] K. Jourde, D. Gibert, J. Marteau, J. de Bremond d'Ars, S. Gardien, C. Girerd, and J.C. Ianigro. Monitoring temporal opacity fluctuations of large structures with muon radiography: a calibration experiment using a water tower. Scientific Reports, 6(23054), 2016.

[3] D. Heck, J. Knapp, J. N. Capdevielle, G. Schatz, and T. Thouw. CORSIKA: a Monte Carlo code to simulate extensive air showers. February 1998.

[4] A. Ferrari, P.R. Sala, Milan /CERN /INFN, A. Fasso, /SLAC, J. Ranft, and /Siegen U. Fluka: A multi-particle transport code. 122005.

[5] S. Agostinelli, J. Allison, K. Amako, J. Apostolakis, , et al. Geant4 - a simulation toolkit. Nuclear Instruments and Methods in Physics Research Section A: Accelerators, Spectrometers, Detectors and Associated Equipment, 506(3):250-303, jul 2003.

[6] I. Sidelnik and the LAGO Collaboration. The sites of the latin american giant observatory. In The 34th International Cosmic Ray Conference, volume PoS(ICRC2015), page 665, 2015.

[7] H. Asorey, S. Dasso, and the LAGO Collaboration. LAGO: the latin american giant observatory. In The 34th International Cosmic Ray Conference, volume PoS(ICRC2015), page 247, 2015.

[8] H. Asorey, L.A. Núñez, and M. Suárez-Durán. Preliminary results from the latin american giant observatory space weather simulation chain. Space Weather, 16(5):461-475, 2018.

[9] A. Letessier-Selvon and T. Stanev. Ultrahigh energy cosmic rays. Rev. Mod. Phys., 83:907-942, Sep 2011.

[10] S. Ostapchenko. Monte Carlo treatment of hadronic interactions in enhanced Pomeron scheme: QGSJET-II model. Physical Review D, 83(1):014018, jan 2011.

[11] F. X. Kneizys, L. W. Abreu, G. P. Anderson, J. H. Chetwynd, et al. The MODTRAN 2/3 report and LOWTRAN 7 model. Technical report, 1996.

[12] D.F. Smart and M.A. Shea. Fifty years of progress in geomagnetic cutoff rigidity determinations. Advances in Space Research, 44(10):1107 - 1123, 2009. Cosmic Rays From Past to Present.

[13] R. Modzelewska and M.V. Alania. The 27-day cosmic ray intensity variations during solar minimum 23/24. Solar Physics, 286(2):593-607, 2013.

[14] J.J. Masías-Meza, S. Dasso, P. Démoulin, L. Rodriguez, and M. Janvier. Superposed epoch study of icme sub-structures near earth and their effects on galactic cosmic rays. Astronomy \& Astrophysics, 592:A118, 2016.

[15] H.V. Cane. Coronal Mass Ejections and Forbush Decreases. Space Science Reviews, 93(1-2):55-77, 2000. 
[16] H. Asorey and the Pierre Auger Collaboration. Measurement of Low Energy Cosmic Radiation with the Water Cherenkov Detector Array of the Pierre Auger Observatory. In Proceedings of the 33th ICRC, pages 41-44, Beijing, China, 2011.

[17] I. Angelov, E. Malamova, and J. Stamenov. The forbush decrease after the gle on 13 december 2006 detected by the muon telescope at beo-moussala. Advances in Space Research, 43(4):504-508, 2009.

[18] S. Dasso, H. Asorey, and Pierre Auger Collaboration. The scaler mode in the pierre auger observatory to study heliospheric modulation of cosmic rays. Advances in Space Research, 49:1563-1569, June 2012.

[19] M.A. Mostafá and for the HAWC Collaboration. The high-altitude water cherenkov observatory. Brazilian Journal of Physics, 44(5):571-580, Oct 2014.

[20] H. Asorey, S. Dasso, L. A. Núñez, Y. Pérez, C. Sarmiento-Cano, M. Suárez-Durán, and the LAGO Collaboration. The LAGO space weather program: Directional geomagnetic effects, background fluence calculations and multi-spectral data analysis. In The 34th International Cosmic Ray Conference, volume PoS(ICRC2015), page 142, 2015.

[21] A. Filevich, P. Bauleo, H. Bianchi, J.R. Martino, and G. Torlasco. Spectral-directional reflectivity of tyvek immersed in water. Nuclear Instruments and Methods in Physics Research Section A: Accelerators, Spectrometers, Detectors and Associated Equipment, 423(1):108-118, 1999.

[22] D. Allard, I. Allekotte, C. Alvarez, H. Asorey, H. Barros, X. Bertou, O. Burgoa, M. Gomez Berisso, O. Martínez, P. Miranda Loza, T. Murrieta, G. Perez, H. Rivera, A. Rovero, O. Saavedra, H. Salazar, J.C. Tello, R. Ticona Peralda, A. Velarde, and L. Villaseñor. Use of water-cherenkov detectors to detect gamma ray bursts at the large aperture GRB observatory (lago). Nuclear Instruments and Methods in Physics Research Section A: Accelerators, Spectrometers, Detectors and Associated Equipment, 595(1):70 - 72, 2008. RICH 2007Proceedings of the Sixth International Workshop on Ring Imaging Cherenkov Detectors.

[23] W. Wang, S. Qian, J. Xia, Z. Ning, Y. Cheng, M. Qi, Y. Heng, Z. Wang, X. Li, S. Liu, and X. Lei. Performance of the 8-in. R5912 photomultiplier tube with super bialkali photocathode. Journal of Instrumentation, 10:T08001, August 2015.

[24] A. Etchegoyen, P. Bauleo, X. Bertou, C.B. Bonifazi, A. Filevich, M.C. Medina, D.G. Melo, A.C. Rovero, A.D. Supanitsky, A. Tamashiro, et al. Muon-track studies in a water cherenkov detector. Nuclear Instruments and Methods in Physics Research Section A: Accelerators, Spectrometers, Detectors and Associated Equipment, 545(3):602-612, 2005.

[25] A. Galindo and the LAGO Collaboration. Sensitivity of LAGO and Calibration of a Water-Cherenkov Detector in Sierra Negra, México. In ICRC 2015 Id. ICRC2015_673, volume ICRC2015, 2015.

[26] A. Galindo et al. Calibration of a large water-cherenkov detector at the sierra negra site of lago. Nuclear Instruments and Methods in Physics Research Section A: Accelerators, Spectrometers, Detectors and Associated Equipment, 861:28 - 37, 2017.

[27] S. Vernetto. Detection of gamma-ray bursts in the 1 gev-1 tev energy range by ground-based experiments. Astroparticle physics, 13(1):75-86, 2000. 